\title{
A Multiscale Computational Model of Calcium-Mediated Ectopy in the Human Postinfarction Heart
}

\author{
Fernando O Campos ${ }^{1}$, Joao F Fernandes ${ }^{1}$, Yohannes Shiferaw ${ }^{2}$, Titus Kuehne ${ }^{3}$, Rodrigo Weber dos \\ Santos $^{4}$, Martin J Bishop ${ }^{1}$, Gernot Plank ${ }^{5}$ \\ ${ }^{1}$ King's College London, London, United Kingdom \\ ${ }^{2}$ California State University, Northridge, CA, United States \\ ${ }^{3}$ German Heart Center Berlin, Berlin, Germany \\ ${ }^{4}$ Federal University of Juiz de Fora, Juiz de Fora, Brazil \\ ${ }^{5}$ Medical University of Graz, Graz, Austria
}

\begin{abstract}
A variety of arrhythmias following myocardial infarction are implicated with ectopic beats (EBs) resulting from spontaneous calcium $\left(\mathrm{Ca}^{2+}\right.$ ) release ( $\mathrm{SCR}$ ) events. However, investigation of these arrhythmias is hampered by the lack of adequate techniques to assess with certainty abnormalities at the subcellular scale and arrhythmogenic events at the organ level. The aim of this study was to construct a multiscale computational model to investigate SCR-mediated ectopy within the infarcted human heart. To achieve this goal, an experimentally based phenomenological model of SCR events was integrated in the equations for $\mathrm{Ca}^{2+}$ cycling of an human ventricular action potential model. This augmented myocyte model was used in in-silico experiments with a postinfarction biventricular (BiV) model constructed based on magnetic resonance imaging data. The infarct scar and border zone (BZ) were segmented by thresholding the voxel intensity within the ventricular wall. These were then used to build a finite element mesh. Within the multiscale human BiV model we show that SCR-mediated DADs in cells interspersed with fibrosis in the infarcted BZ are capable of overcoming local source-sink mismatches to trigger an EB. The results presented here are the first to show that EBs resulting from abnormalities at the subcellular level can be studied using highly detailed human heart models.
\end{abstract}

\section{Introduction}

Ventricular tachycardias (VTs) associated with ischemic heart disease (IHD) are still the major cause of sudden death globally. A series of changes following IHD results in electrophysiological and structural remodelling in the infarcted region setting the stage for VTs [1]. Experimental evidence suggests that a variety of these arrhythmias are attributed to triggered activity due to delayed afterdepolarizations (DADs) in the infarct border zone (BZ) [2]. At the cellular level, DADs have been linked to spontaneous calcium $\left(\mathrm{Ca}^{2+}\right)$ release (SCR) events from the sarcoplasmic reticulum (SR) [3]. At the tissue scale, a triggered action potential (AP) can only capture if the net ionic current generated by DADs is sufficient to overcome local the electrotonic load posed by the surrounding myocardium [4]. However, fibrosis in the BZ has been shown to be capable of reducing electrotonic load favoring ectopy.

The link between abnormal SCR events and ectopy within the ventricles has been a topic of intense research in our group [5,6]. In previous studies we have used simplified as well as anatomically accurate rabbit tissue models to study $\mathrm{Ca}^{2+}$-mediated ectopy $[5,6]$. However, how DADs resulting from subcellular SCR events summate to form EBs in the human heart has not been investigated. In this work we present a multiscale computational model to study SCR-mediated EBs in the human postinfarction heart.

\section{Methods}

\subsection{Phenomenological Model of SCR Events in Human Ventricular Myocytes}

In this work, the ten Tusscher et al. [7] (TT) model was used to simulate ionic membrane dynamics in human ventricular myocytes. Following our previous computational studies [5,6], key parameters of the TT model were modified to increase its propensity for $\mathrm{Ca}^{2+}$-mediated DADs. Specifically, the strength of the electrogenic sodium-calcium exchange current was doubled and the maximum conductance of the inward rectifier potassium current was decreased to $30 \%$ of its control value.

During excitation contraction coupling, $\mathrm{Ca}^{2+}$ is released 
from the $\mathrm{SR}$ in a mechanism known as $\mathrm{Ca}^{2+}$-induced $\mathrm{Ca}^{2+}$ release (CICR). CICR occurs when $\mathrm{Ca}^{2+}$ influx, via the Ltype calcium $\mathrm{Ca}^{2+}$ current $\left(I_{C a L}\right)$, during the course of an AP activates ryanodine receptor (RyR) channels on the SR membrane. In the TT model, CICR is described by the RyR release current $I_{\text {rel }}$ that injects $\mathrm{Ca}^{2+}$ into the subsarcolemmal subspace [7]. However, under certain conditions, $\mathrm{Ca}^{2+}$ release from the $\mathrm{SR}$ can occur in the absence of $I_{C a L}$ [8]. In this case, $\mathrm{Ca}^{2+}$ release is induced by one or a few RyR-channel random openings, which can allow sufficient $\mathrm{Ca}^{2+}$ flux from the SR to initiate CICR. These local release events are referred to as spontaneous $\mathrm{Ca}^{2+}$ sparks and have been reported in $\mathrm{Ca}^{2+}$ overloaded myocytes [3], heart failure [9] as well as in cells that survive in the infarcted heart [2]. The $\mathrm{Ca}^{2+}$ released during a spontaneous spark can also diffuse and lead to $\mathrm{Ca}^{2+}$ waves that propagate across the cell causing a substantial depletion of $\mathrm{Ca}^{2+}$ from the SR. To model these SCR events in human ventricular cells, we write $I_{r e l}$ as a sum:

$$
I_{r e l}=I_{C I C R}+I_{S C R}
$$

where $I_{C I C R}$ is the $\mathrm{Ca}^{2+}$ released into the cell due to CICR as described in the TT model; and $I_{S C R}$ is the $\mathrm{Ca}^{2+}$ released into the cell due to SCR events shown by Chen et al. [8] to satisfy the following differential equation:

$$
\begin{array}{r}
\frac{d I_{S C R}(t)}{d t}=g_{R y R} C a_{S R}(t) R(t)-\frac{I_{S C R}(t)}{T} \\
T=\frac{\tau_{d}}{1-\left(\tau_{d} \frac{d C a_{S R}(t)}{d t}\right)}
\end{array}
$$

where $g_{R y R}$ is the average conductance of a RyR cluster, $C a_{S R}$ is the $\mathrm{Ca}^{2+}$ concentration in the SR and $\tau_{d}$ is the average lifetime of a $\mathrm{Ca}^{2+}$ spark. $I_{S C R}(t)$ can be computed directly from the rate at which $\mathrm{Ca}^{2+}$ sparks occur via $\mathrm{RyR}$ channel fluctuations and/or propagating $\mathrm{Ca}^{2+}$ waves [8].

\subsection{Magnetic Resonance Imaging-based Model of the Human Heart}

Anatomically-detailed models can be generated from segmented magnetic resonance imaging (MRI) data. The conversion of medical images into discrete finite element (FE) models relies upon model generation pipelines such as the one illustrated in Fig. 1. The steps of the pipeline are explained in detail below.

Data from a patient (male, 58 years, weight $80 \mathrm{~kg}$, height $170 \mathrm{~cm}$ ) with IHD was used to build the 3D biventricular (BiV) computer model in this study. Access to the retrospective anonymized data was granted by the Clinical Ethics Committee of the German Heart Center Berlin within the Cardioproof project. The patient underwent MRI in a whole body 1.5 Tesla scanner (Achieva R 3.2.2.0,

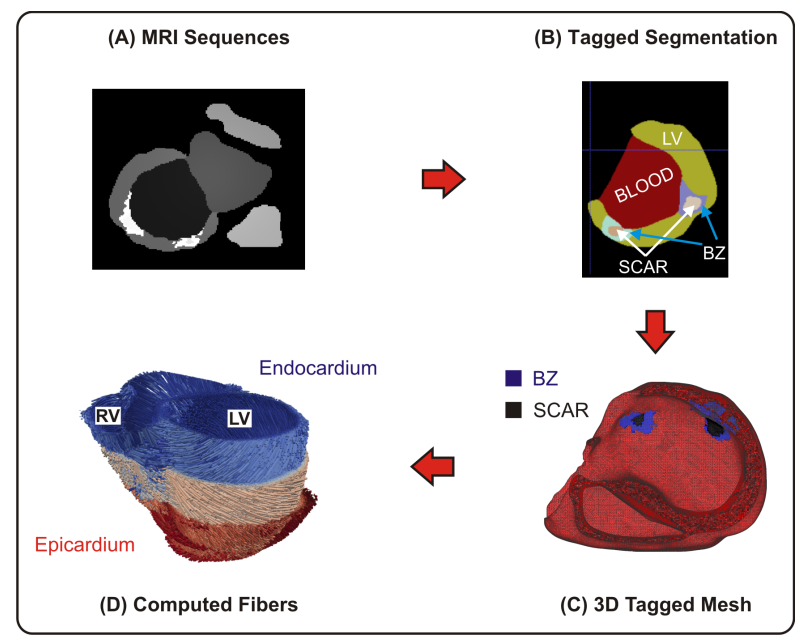

Figure 1. Pipeline of the 3D human heart model generation. A) MRI of a postinfarction human heart. B) Segmented MRI. C) MRI-based tetrahedral finite element mesh. D) Computed fiber orientations.

Philips Medical Systems, Best, The Netherlands) using a five-element cardiac phased-array coil. Three-dimensional anatomy of the ventricles was determined by balanced turbo field echo cine short axis and 3D whole heart.

A 8-label structural segmentation of ventricles, blood pools, atria, aortic root and pulmonary artery was performed via semi-automatic watershed 3D based algorithm embed in ZIBAmira (Zuse Institute Berlin, Germany). Late gadolinium enhanced MRI was used for imaging myocardial infarct, which is subdivided into scar tissue and a BZ by thresholding the images based on the intensity values of the voxels.

A tetrahedral FE mesh was created based on the segmented MRI of the ventricles (Fig. 1B) using Tarantula (CAE Software Solutions, Eggenburg, Austria). Tags from the input segmentation were also mapped onto the generated mesh (Fig. 1C). Myocardial fiber orientations were incorporated into the $\mathrm{BiV}$ model using a rule-based approach [10] (see Fig. 1D).

Fibrosis was modelled by including synthetic patterns of non-conducting tissue in the BZ [6]. The amount of fibrosis in relation to myocardium in the $\mathrm{BZ}$ was $50 \%$ (five different patterns were constructed).

\subsection{Computational Protocols}

Similar to experimental protocols [3], extracellular $\mathrm{Ca}^{2+}\left(\left[\mathrm{Ca}^{2+}\right]_{e}\right)$ in the DAD-prone TT myocyte model was varied from $1.8 \mathrm{mmol} / \mathrm{L}$ to $3.6 \mathrm{mmol} / \mathrm{L}$. The model was then paced at $2.0 \mathrm{~Hz}$ for 100 cycles to stabilize. Pacing was followed by a 2000 -ms pause within which triggered AP formation was recorded. Single-cell model states at the 
end of the pacing protocol were stored and used as initial conditions for the tissue models.

The BiV model was initialized with stabilized singlecell model states and paced at the apex. The stimulus was followed by a 2000-ms pause in order to see whether EBs would emerge. SCR events were inhibited in the myocardium to investigate abnormal triggered activity only in cells in the infarct BZ. Moreover, due to the stochastic nature of the SCR events, $\mathrm{N}=100$ simulations for each set of experiments were performed making a total of 600 simulations.

Electrophysiological activity was simulated within the models using the Cardiac Arrhythmia Research Package (CARP) [11] (http://carp.meduni-graz.at). Throughout, a monodomain representation of tissue electrical dynamics was used with ionic dynamics represented by the augmented TT model.

\subsection{Data Analysis}

The number of simulations in which an EB was observed, $n$, was recorded to compute the probability $\mathrm{p}_{E B}=$ $n / N$. Furthermore, location as well as the waiting time until the onset of an EB, $\mathrm{t}_{E B}$, was determined as the time instant of a triggered AP crossing $-10 \mathrm{mV}$.

\section{Results}

The DAD-prone TT model was paced according to the protocol previously described. Fig. 2 shows two different traces resulting from stochastic SCR events. SCRs led to either a DAD (blue trace) or a triggered AP (red trace).

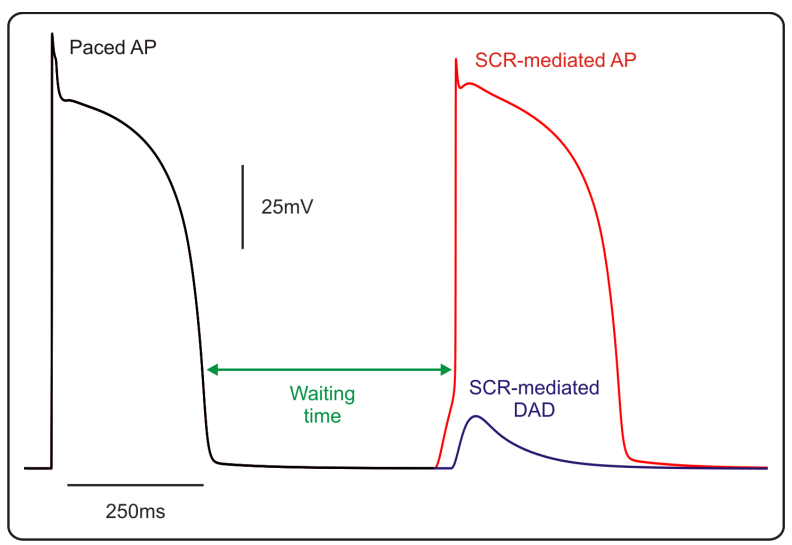

Figure 2. Single-cell results. Subthreshold DAD (blue) and triggered AP (red) resulting from SCR events.

Statistics obtained with the stochastic model are presented in Fig. 3. $\mathrm{p}_{E B}$ increased while $\mathrm{t}_{E B}$ decreases as the cell becomes overloaded with $\mathrm{Ca}^{2+}$. Note that the standard deviation of $\mathrm{t}_{E B}$ also decreases at higher $\left[\mathrm{Ca}^{2+}\right]_{e}$.

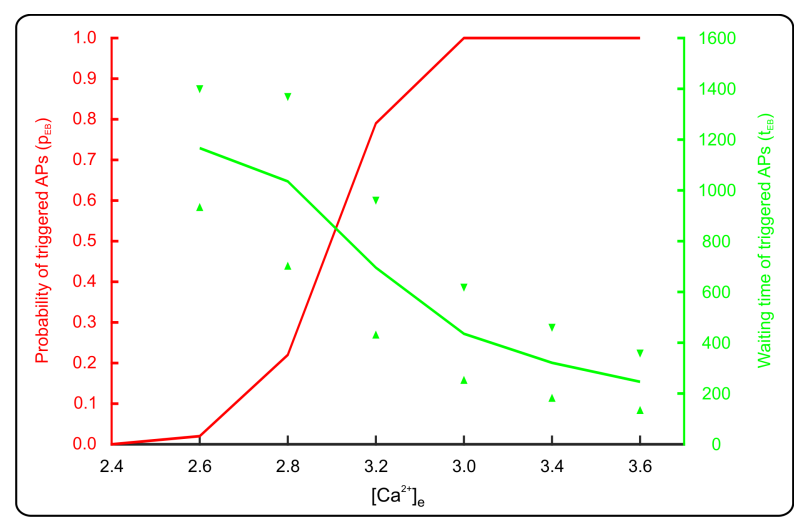

Figure 3. Statistics of SCR-mediated triggered APs in single-cell experiments. Probability $\mathrm{p}_{E B}$ (red) as well as waiting time $\mathrm{t}_{E B}$ (green) of triggered APs as function of $\left[\mathrm{Ca}^{2+}\right]_{e}$ are shown.

SCR events in the BiV model were induced by increasing $\left[\mathrm{Ca}^{2+}\right]_{e}$ to $3.0 \mathrm{mmol} / \mathrm{L}$. Although DADs were observed $(5.75 \mathrm{mV}$ in average), these were not able to overcome the tissue electrotonic load to trigger an EB in any of the $\mathrm{N}=100$ simulations.

In order to investigate if fibrosis can disrupt this protective mechanism and promote EBs, random synthetic fibrosis patterns were added to the $\mathrm{BZ}$ of the $\mathrm{BiV}$ model. As as described in the Methods, $50 \%$ of the myocytes in the BZ were replaced by fibrosis. EBs were observed in $50 \%$ of $\mathrm{N}$ $=500$ simulations ( 100 for each of the 5 random patterns). Spatial distributions of the transmembrane potential of an EB following a paced beat are shown in Fig. 4. DADs were observed around $t=1500 \mathrm{~ms}$ in surviving strands of myocardium. These DADs were capable of summate and trigger an EB at $\mathrm{t}=1570 \mathrm{~ms}$ that entered the healthy myocardium and propagated throughout the ventricles.

\section{Discussion}

In this study we constructed a multiscale computational model to investigate the interplay between SCR-mediated triggered activity and EB formation in the infarcted human heart. An experimentally-based phenomenological model of SCR events was coupled to a state-of-the art ionic model of the human ventricular AP. This novel cell model was incorporated in a $\mathrm{BiV}$ model constructed based on structural MRI from a patient with IHD. Although electrophysiological remodelling was sufficient to induce DADs at the single cell level, electrotonic loading meant that capture did not occur at the full tissue level. However, fibrosis within the BZ disrupted the local electrotonic load making capture possible.

EBs, the first initiating beats of a VT, have been associated to SCRs at the subcellular level. The frequency of 


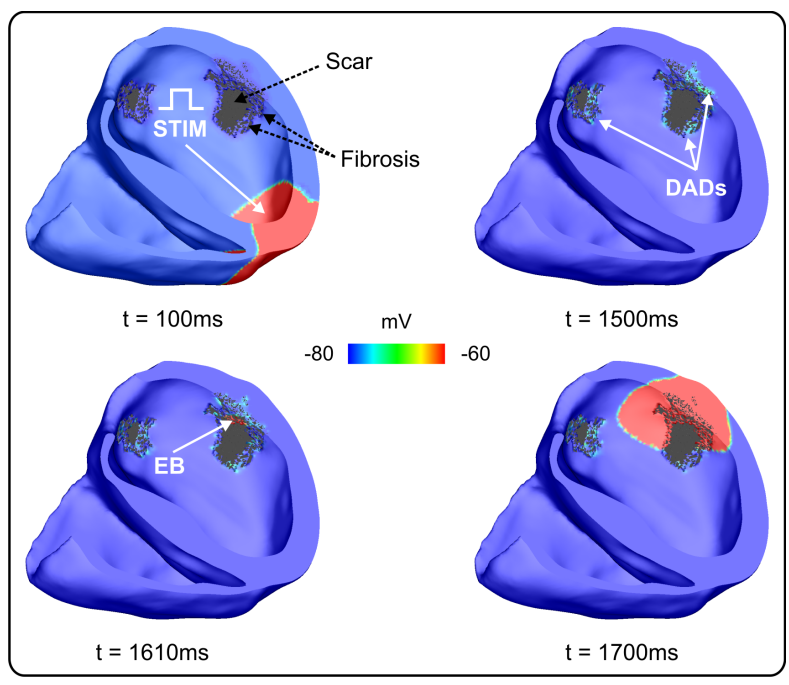

Figure 4. Genesis of a SCR-mediated EB within the BZ.

these aberrant SCR events has been shown to increase with $\mathrm{Ca}^{2+}$ overload in experiments rat ventricular myocytes [3]. Moreover, the time variability of SCRs was shown to decrease as the diastolic SR load increases [3]. Fig. 3 shows that the phenomenological human model qualitatively reproduces this experimental observation.

Although SCRs may induce APs in isolated cells, this is not necessarily the case in coupled tissue due the heart's protective source-sink mismatch [4]. Indeed, no EBs were observed in simulations with the BiV model. Fibrosis has been demonstrated to disrupt this protective mechanism and promote ectopy in in-silico experiments with rabbit hearts [6]. Adding synthetic patterns of fibrosis to the BZ of the human BiV lead to EB formation (see Fig. 4), which is in agreement with our previous findings with idealized and anatomically-detailed rabbit infarct models.

The results presented here are the first to show that $\mathrm{Ca}^{2+}$-mediated EBs can be studied using highly detailed human heart models. The use of human data is one of many steps towards the clinical use of computer models as diagnostic tools for future minimal-invasive diagnosis and treatment of cardiac arrhythmias.

\section{Acknowledgements}

The present work was support by the National Institutes of Health (grant number 1RO1 HL 10119601), the Austrian Science Fund (grant number F3210-N18), CNPq (Conselho Nacional de Desenvolvimento Científico e Tecnológico) - Brazil and the British Heart Foundation through project grant $\mathrm{PG} / 16 / 81 / 32441$.

\section{References}

[1] Nattel S, Maguy A, Le Bouter S, Yeh YH. Arrhythmogenic ion-channel remodeling in the heart: heart failure, myocardial infarction, and atrial fibrillation. Physiol Rev 2007; 87(2):425-56.

[2] Hirose M, Stuyvers BD, Dun W, ter Keurs HE, Boyden PA. Function of $\mathrm{ca}(2+)$ release channels in purkinje cells that survive in the infarcted canine heart: a mechanism for triggered purkinje ectopy. Circ Arrhythm Electrophysiol 2008; 1(5):387-95.

[3] Wasserstrom JA, Shiferaw Y, Chen W, Ramakrishna S, Patel H, Kelly JE, O’Toole MJ, Pappas A, Chirayil N, Bassi $\mathrm{N}$, Akintilo L, Wu M, Arora R, Aistrup GL. Variability in timing of spontaneous calcium release in the intact rat heart is determined by the time course of sarcoplasmic reticulum calcium load. Circ Res 2010;107(9):1117-26.

[4] Xie Y, Sato D, Garfinkel A, Qu Z, Weiss JN. So little source, so much sink: Requirements for afterdepolarizations to propagate in tissue. Biophysical Journal 2010; 99:1408-1415.

[5] Campos FO, Shiferaw Y, Prassl AJ, Boyle PM, Vigmond EJ, Plank G. Stochastic spontaneous calcium release events trigger premature ventricular complexes by overcoming electrotonic load. Cardiovasc Res 2015;107:175-83.

[6] Campos FO, Shiferaw Y, Weber dos Santos R, Plank G, Bishop MJ. Microscopic isthmuses and fibrosis within the border zone of infarcted hearts promote calcium-mediated ectopy and conduction block. Front Phys 2018;6:57.

[7] ten Tusscher KH, Panfilov AV. Alternans and spiral breakup in a human ventricular tissue model. Am J Physiol Heart Circ Physiol 2006;291(3):H1088-H1100.

[8] Chen W, Aistrup G, Wasserstrom JA, Shiferaw Y. A mathematical model of spontaneous calcium release in cardiac myocytes. Am J Physiol Heart Circ Physiol 2011; 300:H1794-H1805.

[9] Hoeker GS, Katra RP, Wilson LD, Plummer BN, Laurita KR. Spontaneous calcium release in tissue from the failing canine heart. Am J Physiol Heart Circ Physiol 2009; 297(4):H1235-42.

[10] Bayer JD, Blake RC, Plank G, Trayanova NA. A novel rule-based algorithm for assigning myocardial fiber orientation to computational heart models. Ann Biomed Eng 2012; 40(10):2243-54.

[11] Vigmond E, Hughes M, Plank G, Leon L. Computational tools for modeling electrical activity in cardiac tissue. J Electrocardiol 2003;36:69-74.

Address for correspondence:

Gernot Plank

Neue Stiftingtalstrae 6 (MC1.D.)/IV, 8010 Graz, Austria gernot.plank@medunigraz.at 